IN BRIEF

$\Rightarrow$ GUT MICROBIOTA

\title{
Frailty associated with gut microbiota
}

Frailty, a useful indicator of overall health decrease, associates with gut microbiota in elderly and care-dependent individuals. Jackson and colleagues have now used a clinical index to quantify frailty in 728 female twins from a younger, community-dwelling cohort, in addition to characterizing microbial $16 \mathrm{~S}$ ribosomal RNA signatures from faecal samples. They found a prominent negative correlation between host frailty and gut microbial diversity, involving specific taxonomic associations, which might ultimately be used for diagnostic surveillence or to improve frailty during ageing.

ORIGINAL ARTICLE Jackson, M. et al. Signatures of early frailty in the gut microbiota. Genome Med. 8, 8 (2016)

\section{$\Rightarrow$ THERAPY}

\section{CT-P13: a safe and effective treatment for IBD}

The monoclonal antibody CT-P13 is a biosimilar of infliximab. Owing to a lack of confidence amongst gastroenterologists in using this drug for patients with IBD, a prospective, multicentre study was conducted to examine the safety, efficacy and immunogenicity of CT-P13 for the treatment of Crohn's disease and ulcerative colitis $(n=210)$. The investigators found that CT-P13 induced and maintained high clinical remission and response rates in IBD. However, patients with previous infliximab exposure showed markedly decreased responses and were more prone to allergic reactions.

ORIGINAL ARTICLE Gecse, K. B. et al. Efficacy and safety of the biosimilar infliximab CT-P13 treatment in inflammatory bowel diseases: A prospective, multicentre, nationwide cohort. J. Crohns Colitis 10,133-140 (2016)

\section{COLORECTAL CANCER}

\section{CRC trends reflect human development}

A new study of the trends and patterns of colorectal cancer (CRC) has shown that incidence and mortality vary up to 10-fold worldwide and correlate with the human development index - used to rank countries by socioeconomic factors. In low and middle-income countries, a rapid increase in CRC was found, possibly reflecting adoption of a more Western lifestyle. In developed countries with the highest rates of CRC, trends are stabilizing or decreasing. Given the expected increase in CRC burden in the future, the researchers conclude that targeted resource-dependent actions are needed, including improved primary prevention and better early detection strategies.

ORIGINAL ARTICLE Arnold, M. et al. Global patterns and trends in colorectal cancer incidence and mortality. Gut http://dx.doi.org/10.1136/gutjnl-2015-310912

\section{$\Rightarrow$ VIRAL HEPATITIS}

\section{A new chimeric model of hepatitis $E$ infection}

To improve our understanding of the mechanisms of hepatitis $E$ virus (HEV) replication, a novel mouse model that is humanized using primary human hepatocytes has been established. The mice were infected with two HEV genotypes (1 and 3), and infection and spreading parameters were monitored in various tissues and cell types. These techniques revealed that HEV virions in faeces were more infective than those from serum, and that HEV genotype 1 spread with faster kinetics. Infected mice were also treated with ribavirin, after which a strong reduction in viraemia was seen, demonstrating the suitability of this model for evaluating preclinical antiviral agents.

ORIGINAL ARTICLE Allweiss, L. et al. Human liver chimeric mice as a new model of chronic hepatitis Evirus infection and preclinical drug evaluation. J. Hepatol. http://dx.doi.org/10.1016/j.jhep.2016.01.011 